\title{
Strategies for Management of Peritoneal Dialysis Patients in Singapore during COVID-19 Pandemic
}

Htay $\underline{\text { Htay, }}{ }^{1,2}{ }_{M B B S,}$ MMED, FRCP, Penelope Maxine PK Wong, ${ }^{3} M B B S, M R C P$, Rui-En Ryan Choo, ${ }^{3} M B B S, M R C P$, Ubaidullah S Dawood, ${ }^{2,4}{ }_{M B B S, F R A C P, F A M S}$, Marjorie Wai Yin Foo, ${ }^{1,2}{ }_{M B C h B, F R C P}$, Mathini Jayaballa,${ }^{1,2}{ }_{M B C h B, F R A C P}$,

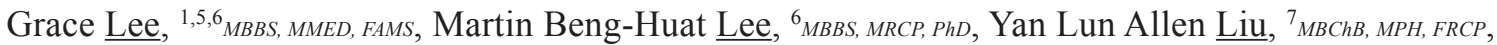
Sanmay Low, ${ }^{8}$ MBBS, MMED, MRCP, Alvin Kok Heong $\underline{\mathrm{Ng}},{ }^{9}{ }_{M B C h B,}$ FRACP, FAMS, Elizabeth Ley Oei, ${ }^{1,2}{ }_{M B C h B}$, Yong Pey $\underline{\text { See }},{ }^{3} M B B S, M R C P$, Rajat Tagore, ${ }^{8}$ MD, FRCPI, MRCP, Yinxia Tai,${ }^{3}$ MBBS,MMED, MRCP, Adrian Liew, ${ }^{10}{ }_{M B B S,}$ FRCP, MClinEpid

\begin{abstract}
Peritoneal dialysis (PD) is the only well-established home-based dialysis therapy in Singapore. As it is a home-based modality, PD should be considered as a preferred mode of kidney replacement therapy (KRT) for patients with kidney failure during this COVID-19 pandemic as it avoids frequent visits to hospitals and/or satellite dialysis centres. The highly infectious nature of this virus has led to the implementation of the Disease Outbreak Response System Condition orange status in Singapore since early February 2020. This paper summarises the strategies for management of several aspects of PD in Singapore during this COVID-19 pandemic, including PD catheter insertion, PD training, home visit and assisted PD, outpatient PD clinic, inpatient management of PD patients with or without COVID-19 infection, PD as KRT for COVID-19 patients with acute kidney injury, management of common complications in PD (peritonitis and fluid overload), and management of PD inventory.
\end{abstract}

Keywords: Home-based dialysis, kidney failure, practice, SARS-CoV-2

Peritoneal dialysis (PD) is the only well-established home-based dialysis therapy in Singapore. As the COVID-19 pandemic may be prolonged, ${ }^{1}$ modification and optimisation of PD patient care to minimise infectious exposure while maintaining consistent patients' outcomes is vital. This paper offers strategies to manage PD patients in Singapore during this current COVID-19 pandemic.

PD catheter insertion. PD catheter insertion is an essential medical service ${ }^{2}$ for patients with kidney failure who opted for PD during this COVID-19 pandemic as it facilitates PD. Nephrologists will need to exercise clinical judgement to determine whether PD initiation can be deferred for kidney failure patients whom themselves, or whose household members are currently under quarantine order $(\mathrm{QO})$ /stay home notice $(\mathrm{SHN})$ or leave of absence (LOA), or patients who have symptoms of acute respiratory tract infection (ARI) or recent history of COVID-19. If such patients require dialysis initiation, PD catheter insertion may proceed under necessary infectious precautions if clinically and logistically feasible.

Where local expertise is available, PD catheters should be placed using the percutaneous Seldinger technique as this procedure can be performed ${ }^{2}$ under sedation and local anaesthesia, and has comparable outcomes to the open and laparoscopic insertion., ${ }^{3,4}$ In the setting of planning $\mathrm{PD}$ for patients with acute kidney injury (AKI), the flexible (rather than rigid) catheter should

\footnotetext{
Department of Renal Medicine, Singapore General Hospital, Singapore

${ }^{2}$ DUKE-NUS Medical School

${ }^{3}$ Department of Renal Medicine,Tan Tock Seng Hospital, Singapore

${ }^{4}$ Department of Renal Medicine, Sengkeng Hospital, Singapore

${ }^{5}$ Gleneagles Hospital, Singapore

${ }^{6}$ Department of Renal Medicine, National University of Singapore, Singapore

${ }^{7}$ Department of Medicine, Khoo Teck Puat Hospital, Singapore

${ }^{8}$ Renal Medicine, Department of Medicine, Ng Teng Fong General Hospital

${ }^{9}$ Department of Renal Medicine, Changi General Hospital

${ }^{10}$ The Kidney \& Transplant Practice Mount Elizabeth Novena Hospital, Singapore

Address for Correspondence: Dr Htay Htay, Department of Renal Medicine, Academia, Level 3, 20 College Road, Singapore General Hospital, Singapore 169856.

Email: htay.htay@singhealth.com.sg
} 
be used and the catheter should be tunnelled to minimise infection. ${ }^{11}$ The catheter must be inserted under strict aseptic conditions preferably via the bedside percutaneous technique or in the operation theatre by trained nephrologists or surgeons in full personal protective equipment (PPE) with prophylactic antibiotics cover. ${ }^{5}$

PD training. While home PD training is encouraged during this COVID-19 pandemic, given the potential shortage of available community nurses to conduct one-to-one training, hospital-based training may be necessary. Patients and accompanying caregivers should be screened telephonically for symptoms of ARI, relevant travel or contact history, and to determine their QO/SHN or LOA status. Patients should be informed to call pre-arrival at the PD centre if they should develop symptoms of ARI.

The total number of patients and accompanying caregivers should be optimised to allow for social distancing in the PD training centre. Training seats in the PD centre should be arranged at least 2 metres apart between patients. ${ }^{6}$ PD trainers, patients and caregivers must wear a mask at all times, practise social distancing, and maintain hand hygiene during the course of PD training. The use of audiovisual PD training materials are encouraged. PD training should be carried out concurrently for patients attending the PD centre for intermittent or urgent-start PD therapy. During the training period, PD nurses should monitor both patients' and caregivers' temperatures.

Formal PD training should be deferred for patients and/or caregivers who are currently under $\mathrm{QO} / \mathrm{SHN} / \mathrm{LOA}$ or in patients with ARI symptoms or confirmed COVID-19. Patients with ARI may proceed with PD training after COVID-19 has been excluded and when asymptomatic. Symptomatic patients who require dialysis initiation, and who have not been excluded for COVID-19 will require hospital admission for PD initiation. Patients admitted for COVID-19 may initiate $\mathrm{PD}$, and complete formal PD training after discharge.

Routine retraining of patients or caregivers should be delayed during the pandemic. Retraining, however, should be considered for situations including necessary change of PD modality, change of caregiver, or PD peritonitis, secondary to improper PD exchange technique. To minimise hospital visits, retraining should take place in the community/patient's home.

Home visit and assisted PD. All routine home visits, including pre-PD home assessment and face-to-face multidisciplinary team meetings post-home visits, should be suspended during the pandemic. Instead, remote monitoring or teleconsultation should be available and home visits conducted only for essential reasons.

The community PD team from National Kidney Foundation (NKF) or PD vendors such as Baxter Healthcare/Fresenius Medical Care or any PD providers in the community should conduct a pre-visit screening before every home visit. The staff who conducts the home visit should be free from symptoms of ARI. Community PD teams should discuss relevant findings and reasons for not conducting the requested home visit to the hospital PD team remotely.

Essential home visits include: the first visit; immediate post-training home visit; home PD training for new patients, patients or caregivers who require further support to ensure safe performance of PD exchanges post-training; essential retraining of existing PD patients; administering of intraperitoneal antibiotics to treat peritonitis for patients unable to self-administer; providing assisted PD for eligible patients; requiring a change of the APD machine due to technical issues; assisting in resetting APD machine for patients unable to reprogramme the machine or who are not on correct, or Claria Sharesource ${ }^{\circledR}$.

Outpatient PD clinic review. PD patients and a maximum of one accompanying caregiver should be screened upon hospital entry, as per local screening workflow. If patients visiting the PD clinic have symptoms of ARI at screening, they should be triaged to the emergency department (ED) for COVID-19 screening and further management. All clinic attendees must wear a mask, practise hand hygiene and social distancing during the clinic visit.

Ad hoc PD clinic reviews should still be available for urgent issues including PD related infections, fluid status that cannot be managed with online monitoring and a potential risk of deterioration without early intervention, dry or wet contamination, as well as catheter dysfunction that does not resolve with laxatives.

The number of PD clinic attendees should be reduced to minimise the risk of infection during the COVID-19 pandemic. Physicians should perform preclinic screening, triaging and reschedule stable PD patients. Hospital pharmacies should arrange home delivery medication services to patients whose appointments have been deferred. Any procedural appointments should be scheduled concurrently with the next clinic visit. PD team should explore teleconsultation for stable PD patients. However, routine PD clinics should be available for patients 
requiring physical review for PD-related issues. Nonessential tests such as Peritoneal Equalibration Test (PET) and $\mathrm{Kt} / \mathrm{V}, 2,7$ and unnecessary alterations of $\mathrm{PD}$ regimen should be avoided. When feasible, patients on Baxter APD system should be switched to Baxter Claria sharesource ${ }^{\circledR}$ system to allow online monitoring and PD therapy titration remotely.

Management of PD for patients admitted to hospital. Haemodynamically stable PD patients may continue on PD therapy. Critically ill PD patients with multi-organ failure and/or requiring other life-support therapy should be temporarily transferred to extracorporeal continuous renal replacement therapy (CRRT).

The PD regimen must be optimised to avoid volume overload in the setting of pneumonia. Patients with COVID-19 should be converted to automated PD (APD) to minimise unnecessary contact of PD nurses with patients. The same APD machine is preferably used for the patient's entire stay in the hospital and must be disinfected before and after each PD therapy as per the hospital's infection control policy. PD nurses must wear full PPE.

In Singapore, effluent disposal follows the hospital's infection control policy. Staff handling the effluent should take extra care to avoid any accidental splash during the discarding of the effluent into the drain or toilet. ${ }^{7}$ Upon discharge, patients and caregivers should be educated on proper disposal of PD effluent, avoiding accidental splash by covering the toilet seat before flushing.

Non-essential admission of PD patients should be deferred during this COVID-19 pandemic. If resources are available, automated PD (APD) is the preferred PD modality for in-patients to minimise patient contact and nursing hours required to perform assisted PD. The same APD machine will be used for the same patient throughout his/her stay in the hospital; however, sharing a machine is allowed only if absolutely necessary and if resources are limited. The APD machine must be disinfected before and after each PD therapy as per the hospital's infection control policy.

Acute PD for patients with AKI and COVID-19. Acute PD can be considered as a modality of CRRT in patients with $\mathrm{AKI} .{ }^{8-10}$ Continuous cycler-assisted peritoneal dialysis is preferred for optimal solute clearance. The dose of PD therapy for patients with COVID-19 and AKI should be adjusted based on the patients' clinical and biochemical needs rather than measured $\mathrm{Kt} / \mathrm{V}_{\text {urea, }}$, which is not recommended during the pandemic. PD can be initiated soon after PD catheter insertion, initially with lower fill volumes of
$1.2 \mathrm{~L}$ to $1.5 \mathrm{~L}$, and gradually increased up to $2 \mathrm{~L}$ if there is no dialysate leak. Acute PD patients should be monitored for electrolyte abnormalities, particularly of hypokalaemia and hyperglycaemia, and also for signs of peritonitis.

Management of common complications in PD patients during pandemic.PD patients presenting with symptoms suggestive of peritonitis should be investigated and managed as outpatients unless there are clinical indications for admission, e.g. suspected acute abdomen (secondary peritonitis), haemodynamically unstable patients, elderly patients who stay alone and cannot reliably self-administer IP antibiotics, concomitant exit-site/tunnel infection, and peritonitis that warrants catheter removal.

Patients who have ARI symptoms and develop peritonitis should be advised to go to the ED for further management. If they are under $\mathrm{QO} / \mathrm{SHN}$, they are to contact their officer-in-charge to arrange for a visit to the ED. PD patients with COVID-19 who develop peritonitis will be managed as inpatients. Mild fluid overload may be managed with teleconsultation and remotely monitored. However, dyspnoeic patients should be advised to go to the ED given that shortness of breath may be due to fluid overload or viral infection. They should be screened for COVID-19 and admitted for further management of their breathlessness. Patients with mild fluid overload and symptoms of ARI (without breathlessness) should be advised to attend a clinic which has facilities to screen for COVID-19 .

PD supply chain and inventory. Lockdown policies of many countries during this COVID-19 pandemic poses a threat to the supply of dialysis fluid/consumables and PD machines. Hospital PD teams should work with local PD suppliers to ensure the delivery of PD solutions and consumables to patients and hospitals without disruption. Patients should be provided with a minimum of 2 weeks supply of PD solutions at home. ${ }^{7}$ In addition, clinicians and multidisciplinary teams within hospitals should monitor their stocks of PD consumables to ensure adequate supply. Stockpiling should be avoided to prevent shortages at individual hospitals, and inter-institution loan in exigencies encouraged.

Publications to optimise the management of PD patients globally during this COVID-19 pandemic are scarce. This is the first paper summarising the strategies for management of several aspects of PD in Singapore and most are opinion-based rather than evidence-based given the paucity of available evidence. Nonetheless, these strategies serve as a guide for physicians 
or nephrologists dealing with PD patients during COVID-19 pandemic in Singapore.

\section{Acknowledgements}

The authors would like to thank A/Prof Lina Choong Hui Lin and Dr Jason Choo Chon Jun from Singapore Society of Nephrology for their support

\section{REFERENCES}

1. Kissler SM, Tedijanto C, Goldstein E, et al. Projecting the transmission dynamics of SARS-CoV-2 through the postpandemic period. Science 2020;368:860-8.

2. Wilkie M, Davies S. Peritoneal Dialysis in the time of COVID-19. Perit Dial Int 2020;40:357-8.

3. Henderson S, Brown E, Levy J. Safety and efficacy of percutaneous insertion of peritoneal dialysis catheters under sedation and local anaesthetic. Nephrol Dial Transplant 2009;24:3499-504.
4. Medani S, Shantier M, Hussein W, et al. A Comparative Analysis of Percutaneous and Open Surgical Techniques for Peritoneal Catheter Placement. Perit Dial Int 2012;32:628-35.

5. Szeto CC, Li PKT, Johnson DW, et al. ISPD Catheter-Related Infection Recommendations: 2017 Update. Perit Dial Int 2017;37:141-54.

6. Social Distancing, Quarantine, and Isolation. Available at: https://www.cdc.gov/coronavirus/2019-ncov/prevent-getting-sick/ social-distancing.html. Updated 17 November 2020. Accessed on 26 April 2020.

7. Strategies regarding COVID-19 in PD patients - International Society for Peritoneal Dialysis. Available at: https://ispd.org/strategiescovid19/. Accessed on 19 April 2020.

8. Ponce D, Berbel MN, Regina de Goes C, et al. High-volume peritoneal dialysis in acute kidney injury: indications and limitations. Clin J Am Soc Nephrol 2012;7:887-94.

9. Cullis B, Abdelraheem M, Abrahams G, et al. Peritoneal dialysis for acute kidney injury. Perit Dial Int 2014;34:494-517.

10. Chionh CY, Ronco C, Finkelstein FO, et al. Acute peritoneal dialysis: what is the "adequate" dose for acute kidney injury? Nephrol Dial Transplant 2010;25:3155-60. 